\title{
CORRECTION
}

\section{Correction to: Abstracts Presented at the Neurocritical Care Society (NCS) 15th Annual Meeting}

C 2018 Springer Science+Business Media, LLC, part of Springer Nature and Neurocritical Care Society

Correction to: Neurocritical Care (2017) 27:S1-S491 https://doi.org/10.1007/s12028-017-0465-9

Assessment of Complications from Decompressive Hemicraniectomy

Error: author excluded from original submission

Correction: Addition of Author: George Lopez, Rush University, Chicago, Illinois

Race/Ethnicity Influences Outcomes in Young Adults with Intracerebral Hemorrhage

Error: authors listed in incorrect order in original submission
Correction: Author 1 should be: Guido Falcone, Department of Neurology, Yale School of Medicine, New Haven, CT

Use of Medical Simulation to Create an Optic Nerve Sheath Diameter (ONSD) Ultrasound Model for Education

Error: author excluded from original submission

Correction: Addition of 1st Author: Heidi M. Felix, Mayo Clinic, Jacksonville, Florida, USA

Published online: 27 July 2018

The online version of the original article can be found under doi: https://doi. org/10.1007/s12028-017-0465-9.

\section{Springer}

